\title{
Application Framework Development for Algorithm Design of PAPR Reduction in OFDM
}

\author{
Mohammed Zakee Ahmed, Ajij D. Sayyad
}

\begin{abstract}
Orthogonal Frequency Division Multiplexing (OFDM) is a well-built candidate for Physical Layer of $5 G$ Communications as like it was there in $3 G$ and $4 G$. With many advantages OFDM has some limitations like synchronization, Peak to Average Power Ratio (PAPR) etc. PAPR in OFDM remained the hot topic in $\mathrm{PHY}$ design of modern wireless communication systems since decades. Researchers are working to solve this problem with various approaches. Being one of the problem solvers team we realized that for a new researcher major effort goes into development of framework rather than actual PAPR algorithm. With this paper we tried to solve this issue by designing a simplified framework for developing, testing and measurement of PAPR of OFDM in Laboratory Virtual Instrumentation Engineering Workbench (LabVIEW) Platform. LabVIEW is a cutting edge, state of the art graphical programming environment which makes programming more simplified by adapting icons and connectors instead of text instructions, which makes computer programming language least complex, so that one can focus more on algorithm design rather than solving syntactical issues of the programming language. There are numerous applications of LabVIEW platform such as Instrumentation, image processing, digital signal processing, digital communication and many more. We have used core programming, signal processing and digital communication modules of LabVIEW for this research work. This paper has three sections, in section one, we explained need of framework, in second section we have explained detailed explanation of framework and its deployment and in the third section result and conclusion has been described..
\end{abstract}

Keywords: PAPR, OFDM, 5G, LabVIEW, NI-USRP 2922

\section{INTRODUCTION}

OFDM is a strong, rugged and multipath fading tolerant modulation/multiplexing technique, which can easily be found in all type of networks including WPAN, WLAN and WMAN/WWAN. It has been adapted in several wireless standard deployments such as Digital Audio Broadcasting: DAB or EUREKA, also in DAB+, Digital Radio Mondale, HD Radio, T-DMB and ISDBTSB, Terrestrial Digital Video Broadcasting: DVB-T and ISDBT, Mobile TV: DVB-H, T-DMB and ISDB-T, Wireless PAN: ultra-wideband (UWB)

Revised Manuscript Received on December 30, 2019.

* Correspondence Author

Mohammed Zakee Ahmed, Assistant professor, Department of Electronics and Telecommunication Engineering, Pune Institute of Computer Technology Pune, India.

Dr. Ajij D Sayyad, Associate Professor, Department of Electronics and Telecommunication Engineering, MIT Aurangabad, India.

(C) The Authors. Published by Blue Eyes Intelligence Engineering and Sciences Publication (BEIESP). This is an open access article under the CC BY-NC-ND license (http://creativecommons.org/licenses/by-nc-nd/4.0/)
IEEE 802.15.3a, Wireless LAN: IEEE 802.11a, g, $\mathrm{n}$ and HIPERLAN/2, Wireless MAN: broadband wireless access standard, IEEE 802.16e, the mobile broadband wireless access standard IEEE 802.20, [1] [2] 4G Long Term Evolution (LTE): Evolved UMTS, Terrestrial Radio Access (E-UTRA). And now it has been considered as a strong candidate for 5G mobile phone standards [3] [4].

OFDM can transmit bulk of data over Radio waves and it is one of the most known prominent multicarrier multiplexing accesses Technique [5]. Mathematically OFDM is expressed as equation (i)

$$
x(t)=\sum_{k=0}^{N-1} X_{k} e^{j 2 \Pi k t / T} \quad 0 \leq \mathrm{t} \leq \mathrm{T}
$$

In proposed method the PAPR block at transmitter and receiver are isolated from other blocks and once a framework is designed, it may be developed exclusively. For framework, a wireless standard may be chosen or even more generalized framework can also be designed, where according to the wireless standard number of sub-carriers, zero padding, reference symbols, modulation scheme and other essentials can be chosen as per the need.

PAPR in OFDM:

The high PAPR shows up when a large number of subcarrier get summed-up together creating large peaks compared to single carrier systems [3]. It has noise like amplitude, with a very large dynamic range and it is more sensitive to carrier frequency offset and drift as compared to single carrier systems Mathematically it can be defined as follows in equation (ii) and (iii).

$$
\begin{gathered}
P A P R=\frac{\max |x(t)|^{2}}{E\left[|x(t)|^{2}\right]} \\
P A P R_{d B}=10 \log _{10}(P A P R)
\end{gathered}
$$

High PAPR increases power consumption of High Power Amplifier (HPA) of transmitter as the high peaks go into the saturation region of the HPA, making non-linearity in signal amplification. This creates in-band distortion and out-off-band distortion resulting increased BER at the receiver and spectral re-growth respectively. This effect not only increases the power consumption of HPA but also increases complexity at ADC and DAC [6] [7] [8]. Choosing wide band power amplifier increases cost of system and it will be waste of cost as high peak in signal is probable event may occur less frequently. 
The PARP value is proportional to number of subcarriers equation (iv) shows value of PAPR is function of $\mathrm{N}$ subcarriers

$$
P A P R_{d B}=10 \log _{10}(N)
$$

For instance if number of subcarriers are 52, as like in IEEE 802.11a/g the instantaneous

PAPR value may be expected as much as $17 \mathrm{~dB}$, which is most unlikely event. Actual instantaneous PAPR goes up to $09 \mathrm{~dB}$ for this standard. By reducing PAPR in OFDM Transmission Power Amplifier Efficiency Improves, signal distortion \& BER can be reduced, by reducing $1 \mathrm{~dB}$ of PAPR approximately $6-9 \%$ transmission power can be minimized [9]. These reasons lead a strong motivation to reduce the PAPR further in OFDM systems. Present methods are good but not best.The complementary cumulative distribution function (CCDF) is used to know how often the random variable- here PAPR, is above a particular level. CCDF computes the power from a time domain signal it is also known as tail distribution or exceedance. The CCDF curve shows the amount of time a signal spends above the average power level of the measured signal, or equivalently, the probability that the signal power will be above the average power level. Performance of transmitted signal is evaluated with CCDF tool for different PAPRs of the signal [10]. The energy per bit to noise power spectral density ratio is evaluation tool of normalized signal to noise ratio measure at receiver side with various channel models like AWGN etc. Here after developing a PAPR reduction scheme we may check its performance at receiver side with BER Vs $\mathrm{E}_{\mathrm{b}} / \mathrm{N}_{0}$ tool. Thus the framework must have data source in binary form, or random sequence generator, various digital modulation and demodulation algorithm, coding and decoding techniques, measurement tools like PAPR measurement, BER Vs $E_{b} / N_{0}$, and flexibility to add, remove and edit PAPR reduction technique.

The algorithm developer should have thorough knowledge of OFDM signal construction of various wireless standards and core LabVIEW programming, then he can easily apply his research fundamentals to design and develop PAPR reduction technique of given mathematical model. In next two consecutive sections, implementation of framework and testing of various popular PAPR reduction techniques (summarized by [1]) have been explained respectively.

\section{PROPOSED WORK: FRAMEWORK DEPLOYMENT}

In proposed work the WLAN standard IEEE 802.11a/g PHY design specification has been referred for OFDM signal design and implementation in LabVIEW Platform. According to these specifications the series of bits is converted into bunch of bits called as symbols, this achieved by one of the four digital modulation techniques i.e. BPSK, 4-QAM, 16-QAM or 64-QAM. The relation of symbol and bits is briefed with equation (v) and (vi).

$$
\begin{aligned}
& M=2^{k} \\
& T=k T_{b}
\end{aligned}
$$

Where,

M-Symbol Level

K-number of bits per symbol T-Symbol Period $\mathrm{T}_{\mathrm{b}}$-bit period
Value of $\mathrm{k}$ will be 1, 2, 4 and 6 for BPSK, 4-QAM, 16-QAM and 64 QAM respectively [11]

\section{a) Block Diagram and Flow Chart}

Figure 1 shows detailed block diagram and flow chart sequence of proposed work. Here the PAPR reduction scheme at transmitter and its counter scheme to recover original signal at receiver is made isolated from other blocks so any one can easily test his algorithm with said scheme.

The following block diagram is designed with following configuration. The OFDM symbol structure consists of 52 subcarriers out of which 48 are data subcarriers 4 are pilot subcarriers which is known sequence by receiver used for channel estimation. This will help to chose appropriate modulation technique based on estimated channel. Total 12 zeros are padded at upper and lower end to reduce adjacent channel interference. These 64 symbols are transformed with 64 IFFT. Last 8 symbols are prefixed making 72 symbol in one OFDM frame, adding this prefix is called as Cyclic Prefix (CP) [12][13].

\section{b) Algorithm}

- Get binary Data from source

- Apply digital modulation and convert bits into Symbols

- Map Serial sequence of Symbols to Parallel Chunks

- Apply PAPR algorithm under test

- Join parallel chunks making serial sequence of symbols

- Add Cyclic prefix

- Handover to Carrier modulation and transmit

- Apply reverse steps at receiver to get original transmitted Binary Data

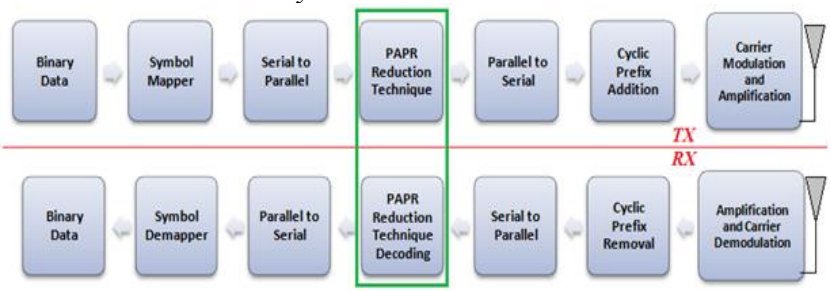

Figure 1: OFDM Transmitter and Receiver Framework with custom amend PAPR reduction Block

Figure 2 shows cited specifications of WLAN IEEE 802.11 standard in LabVIEW. The Various LabVIEW functions called as Virtual Instruments (VI's) are developed and deployed here. Starting from left the first VI is to generate source Data which is here PN Sequence. Later digital modulation of given choice (BPSK, QPSK, 16-QAM and 64-QAM) is applied in S:P block. The next part shows a for loop where the zero padding, pilot insertion is applied. After that the IFFT operation is done with LabVIEW built-in function. Finally CP insertion is performed and signal is analyzed. Figure 3 shows the application framework where we can test any given algorithm for PAPR reduction. The developed PAPR reduction algorithm can be placed as one of the case of case structure in for loop. By making appropriate flow control of code comparative analysis can be done with existing algorithms. Further this framework can be made universal for any given standard. Thus it may be useful in even designing new standard and evaluating it. The receiver design consists of reverse steps of transmitter that is first the $\mathrm{CP}$ will be removed, FFT operation will be performed after that zero padding and pilot subcarriers are removed and finally de-mapping (demodulation) of symbols will give original bit sequence, which can be compared with original sequence to estimate BER. 
Over changing magnitude of noise BER Vs Eb/N0 can be plotted.

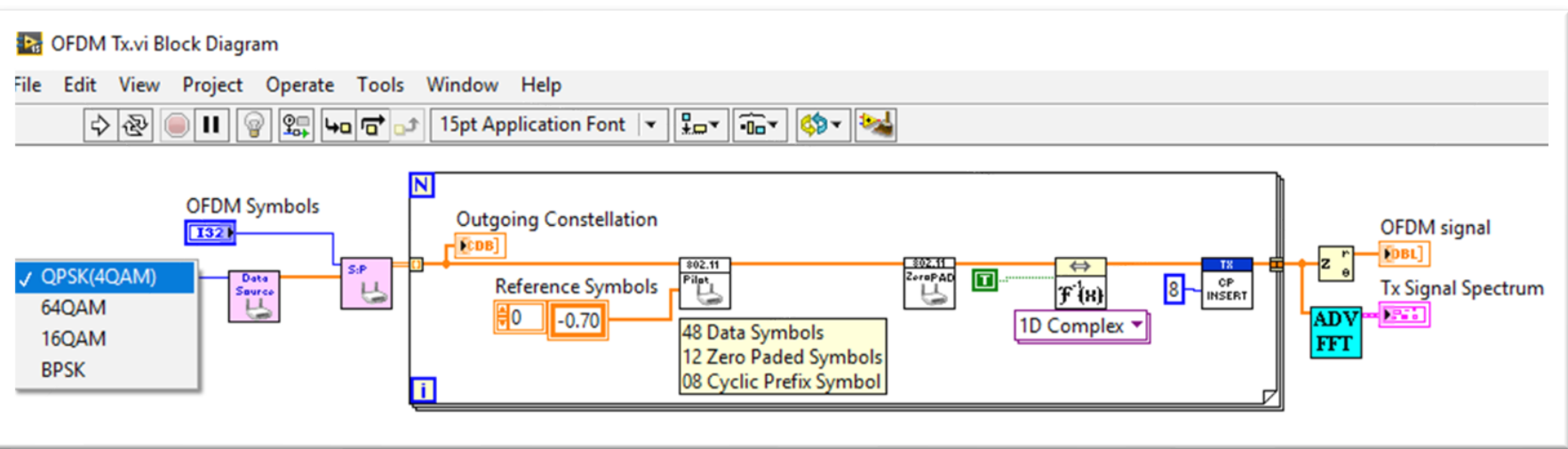

Figure 2: Construction of OFDM signal for IEEE 802.11a WLAN specifications

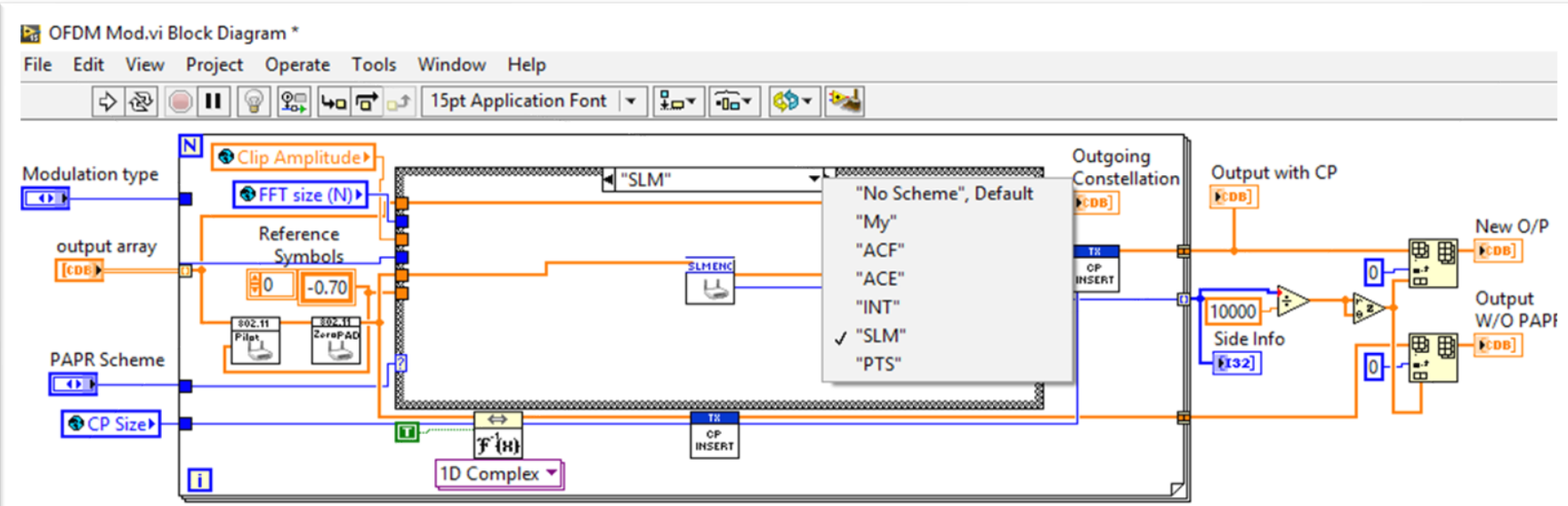

Figure 3: Application Framework for testing PAPR algorithm for IEEE 802.11a WLAN specifications

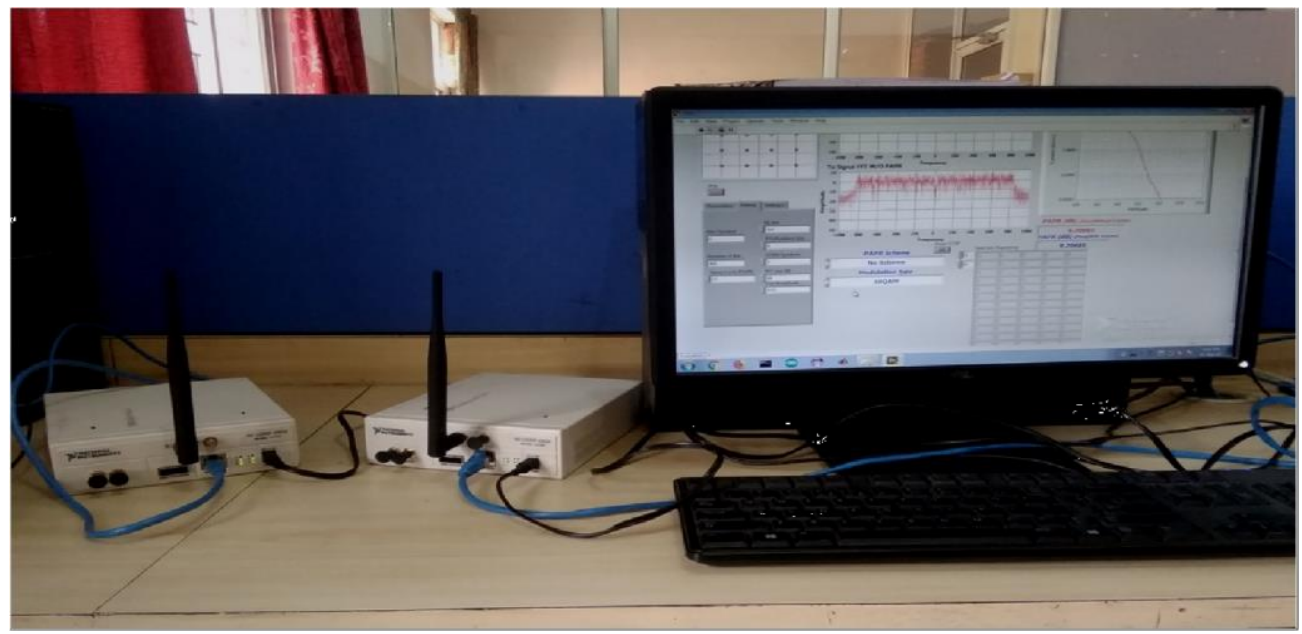

Figure 4: Test Bed with Software Defined Radio NI-USRP 2922

The proposed Framework is executed as computer simulation program in LabVIEW but it can be as it is deployed in real world environment easily, which require a Software Defined Radio platform. NI-USRP is one of the universal software defined radio peripheral which supports execution of LabVIEW program. Figure 4 shows the test bed setup for proposed framework. Here two USRPs have been used as transmitter and receiver, third USRP may be added to add channel as well. In previous section the transmitter and receiver programs were inter-connected with one of the data transmission feature called as queue of LabVIEW, here queue is replaced with NI-USRP pellets.

\section{RESULT ANALYSIS}

The framework with various PAPR reduction techniques is tested for IEEE 802.11a WLAN PHY signal standard specifications. Comparison of various techniques is done with pure OFDM signal i.e. with no-PAPR reduction technique. It is concluded that any PAPR reduction technique can easily be added and comparative analysis can be done with least program development complexity. Developer can focus fully on PAPR reduction technique. 
Figure 5 is the CCDF Analysis of various existing PAPR reduction techniques for BPSK Modulation where PAPR likelihood varies from 6-10 dB.

Further the CCDF Analysis of these PAPR reduction techniques for 4-QAM, 16-QAM and 64-QAM modulation techniques is done and shown in Figure 6,7 and 8 respectively. Here also it can be easily observed the PAPR varies from $6-10 \mathrm{~dB}$, summary is given as in table 1.This work can be further extended for various other standards like DVB, WiMAX, LTE etc. also use of hardware like NI-USRP will help to truly validate the results in real environment

Table 1: Comparative analysis of PAPR values

\begin{tabular}{|c|c|c|c|c|}
\hline \multirow{2}{*}{$\begin{array}{c}\text { PAPR } \\
\text { SCHEMES }\end{array}$} & \multicolumn{4}{|c|}{ PAPR Value (Max) in dB) } \\
\cline { 2 - 5 } & BPSK & 4-QAM & $\begin{array}{c}\text { 16-QA } \\
\text { M }\end{array}$ & $\begin{array}{c}64-Q A \\
\text { M }\end{array}$ \\
\hline No Scheme & 9.6 & 9.4 & 8.9 & 9.8 \\
\hline SLM & 6.5 & 6.7 & 6.6 & 6.8 \\
\hline PTS & 6.8 & 6.2 & 6.3 & 6.6 \\
\hline ACF & 6.7 & 6.6 & 6.3 & 7.4 \\
\hline INT & 8.9 & 6.3 & 7.2 & 7 \\
\hline ACE & 6.7 & 6.9 & 6.4 & 7.9 \\
\hline
\end{tabular}

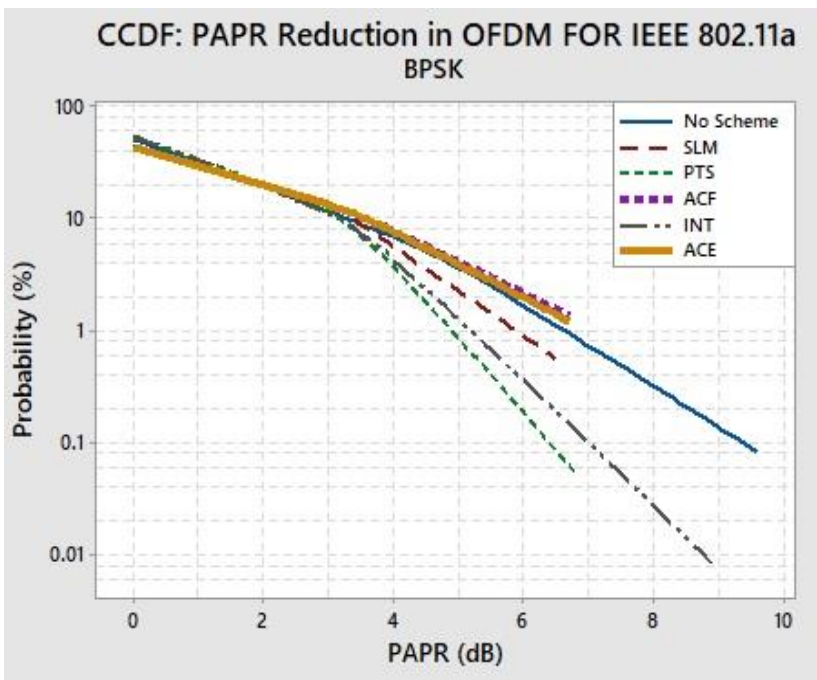

Figure 5: CCDF Analysis of various existing PAPR reduction techniques for BPSK Modulation

\section{CCDF: PAPR Reduction in OFDM FOR IEEE 802.11a} 4-QAM

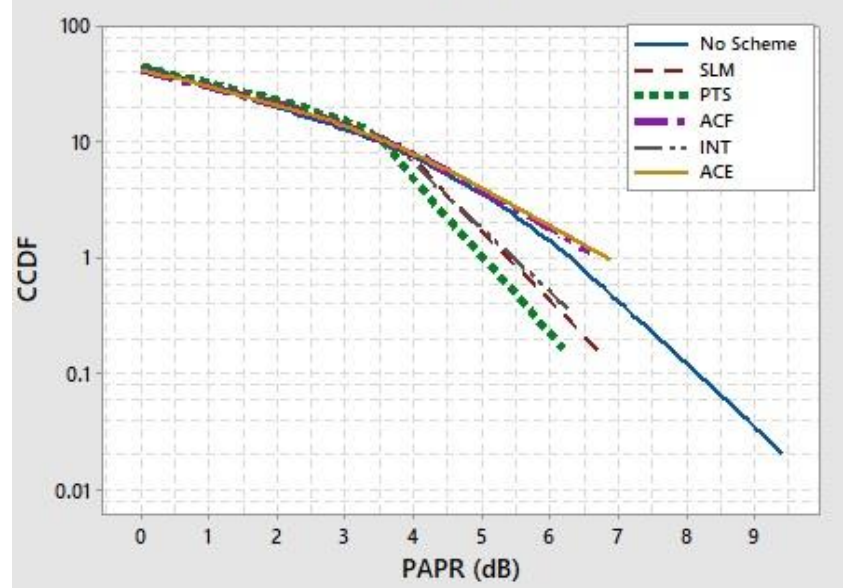

Figure 6: CCDF Analysis of various existing PAPR reduction techniques for 4-QAM Modulation

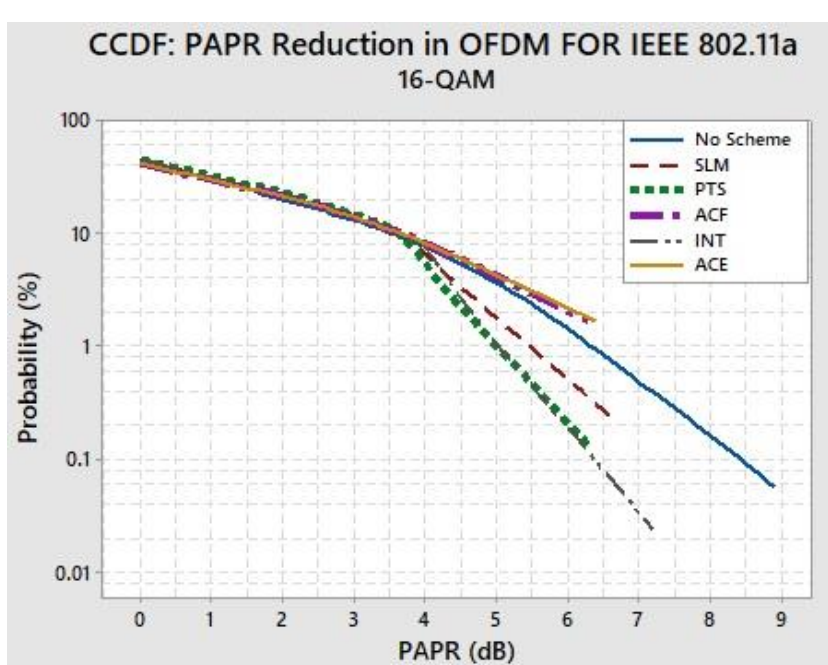

Figure 7: CCDF Analysis of various existing PAPR reduction techniques for 16-QAM Modulation

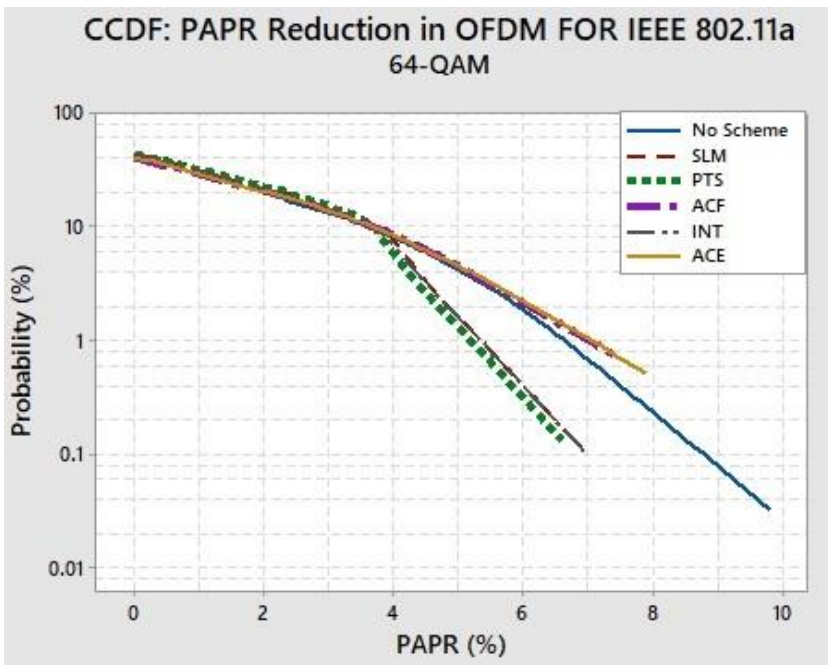

Figure 8: CCDF Analysis of various existing PAPR reduction techniques for 64-QAM Modulation

\section{CONCLUSION}

We have successfully designed and implemented a simplified Application framework to develop and test PAPR reduction algorithm using LabVIEW platform. The framework is tested for various existing PAPR reduction techniques viz. Amplitude Clipping and Filtering (ACF), Interleaving (INT), Active Constellation Extension (ACE), Selective Mapping (SLM) and Partial Transmit Sequence (PTS). Both software and hardware simulations are done to test the reliability of proposed algorithm.

\section{ACKNOWLEDGMENT}

Authors would like to present a greater gratitude to Principal PICT and HOED for their motivation and Support, also Pune Institute of Computer Technology for providing resources and research nurturing environment. 


\section{REFERENCES}

1. Ritu Kumari, Mridul Chawla, "Review of PAPR Reduction Techniques for 5G System", International Journal of Electronics and Communication Engineering. ISSN 0974-2166 Volume 10, Number 1 (2017), pp. 35-44.

2. GSMA Intelligence, "Understanding 5G: Perspectives on future technological advancements in mobile," White paper, 2014.

3. H. Holma, A. Toskala, and J. Reunanen, LTE Small Cell Optimization: 3GPP Evolution to Release 13. Hoboken, NJ, USA: Wiley, 2015.

4. P. Yang et al., "Design Guidelines for Spatial Modulation," IEEE Commun.Surveys \& Tutorials, vol. 17, no. 1, 1st qtr. 2015, pp. 6-26.

5. Mathieu Van Eeckhaute, André Bourdoux, Philippe De Doncker, François Horlin, 'Performance of emerging multi-carrier waveforms for $5 \mathrm{G}$ asynchronous communications', Eeckhaute et al. EURASIP Journal on Wireless Communications and Networking (2017) 2017:29 DOI 10.1186/s13638-017-0812-8

6. A Maltsev, A Lomayev, A Khoryaev, A Sevastyanov, R Maslennikov, in 7th IEEE Consumer Communications and Networking Conference. Comparison of Power Amplifier Non-Linearity Impact on $60 \mathrm{GHz}$ Single Carrier and OFDM Systems, (2010), pp. 1-5. doi:10.1109/CCNC.2010.5421601

7. T Wild, F Schaich, in IEEE 81st Vehicular Technology Conference. A Reduced Complexity Transmitter for UF-OFDM, (2015), pp. 1-6. doi:10.1109/VTCSpring.2015.7145643

8. G. Rashwan, S. Kenshi and M. Matin, "Analysis of PAPR hybrid reduction technique based on PTS and SLM," 2017 IEEE 7th Annual Computing and Communication Workshop and Conference (CCWC), Las Vegas, NV, 2017, pp. 1-4. doi: 10.1109/CCWC.2017.7868482.

9. K. Lee, Y. Cho, J. No and D. Lim, "A low-complexity PTS scheme using adaptive selection of dominant time-domain samples in OFDM systems," 2016 URSI Asia-Pacific Radio Science Conference (URSI AP-RASC), Seoul, 2016, pp. 1897-1900. doi: 10.1109/URSIAP-RASC.2016.7601212

10. S. Ku, "An improved low-complexity PTS scheme for PAPR reduction in OFDM systems," 2016 IEEE International Conference on Signal Processing, Communications and Computing (ICSPCC), Hong Kong, 2016, pp. 1-5. doi: 10.1109/ICSPCC.2016.7753598

11. Yomo, Hiroyuki \& Nguyen, Huan \& Kyritsi, Persefoni \& Duc Nguyen, Tien \& S. Chakraborty, Shyam \& Prasad, Ramjee. (2015). PHY and MAC performance evaluation of IEEE 802.11a WLAN over fading channels. IETE Journal of Research. 51. 10.1080/03772063.2005.11416381.

12. Z. He, L. Zhou, Y. Chen and X. Ling, "Low-Complexity PTS Scheme for PAPR Reduction in FBMC-OQAM Systems," in IEEE Communications Letters, vol. 22, no. 11, pp. 2322-2325, Nov. 2018. doi: 10.1109/LCOMM.2018.2871263

13. D. C. Park and S. C. Kim, "Partial Transmit Sequence Scheme for Envelope Fluctuation Reduction in OFDMA Uplink Systems," in IEEE Communications Letters, vol. 22, no. 8, pp. 1652-1655, Aug. 2018.doi: 10.1109/LCOMM.2018.2840542

\section{AUTHORS PROFILE}

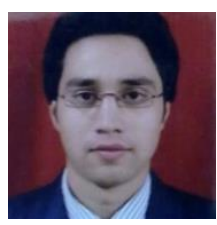

Mohammed Zakee Ahmed, accomplished his M-Tech from National Institute of Electronics \& Information Technology (NIELIT),(erstwhile DOEACC Society), an Autonomous Scientific Society under the administrative control of Ministry of Electronics \& Information Technology (MoE\&IT), Government of India, has nine years of experience in teaching and research. He has qualified Certified LabVIEW Associate Developer (2015). Presently working as Assistant professor in department of Electronics and Telecommunication Engineering at Pune Institute of Computer Technology Pune

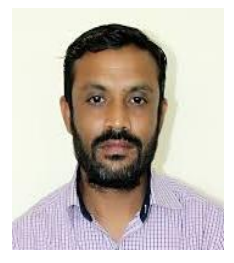

Dr. Sayyad Ajij has accomplished his PhD (Systems and Control Engineering) from IIT Bombay Mumbai, has more than 12 years of teaching and research experience. Presently he is working as Associate Professor and Vice Principal (Academics) at Marathwada Institute Of Technology, Aurangabad his Area of Specialization is Industrial Automation, Robotics and Wireless Communication 